\title{
Critical Analysis of Bone Marrow Studies: A 5 Year Experience
}

\author{
Jerusha Samuela Jacob, Febe Renjitha Suman*, Sai Shalini C.N, Rithika Rajendran, Ravi Teja J and Jesu Magdalene S. \\ Department of Pathology, Sri Ramachandra Medical College \& Research Institute, Chennai, Tamilnadu, India
}

\begin{abstract}
Introduction: Bone marrow Aspirate (BMA) and bone marrow trephine biopsy (BMB) are the two diagnostic procedures done to evaluate various hematologic and non-hematologic conditions. Though both the procedures done on the same day are complementary to each other, discrepancies do occur. In few instances aspirate alone is done without a biopsy. This study is aimed to statistically analyze the diagnostic value of both the procedures and the lacuna observed.

Methods: A retrospective laboratory record based analysis was done on the bone marrow investigations reported during a period of 5 years (Jan.2011 - Dec.2015). The bone marrow investigations with simultaneous BMA and BMB done during the period of study were included. Descriptive statistical analysis was done for correlation of BMA and BMB diagnosis.

Results: Aspirate and biopsy were done on 934 cases. The diagnostic sensitivity of BMB was $94.74 \%$ and BMA was $86.14 \%$. BMB and BMA were complementary to each other in $53.21 \%$ cases. BMB alone was diagnostic in $33.61 \%$ cases and BMA alone was diagnostic in $9.31 \%$ cases. Inadequate material for diagnosis was noted in $8.35 \%$ of BMB cases and $27.41 \%$ of BMA cases. Good positive correlation was noted in cases of immune thrombocytopenic purpura, multiple myeloma, anemia, reactive marrow and chronic lymphocytic leukemia.

Conclusion: Though both the procedures were complimentary to each other, inadequate aspirates due to disease conditions and faulty techniques were major drawbacks. It is preferable to perform both the procedures simultaneously for a more conclusive diagnosis.
\end{abstract}

Keywords: Bone Marrow Studies, Bone Marrow Aspirate, Bone Marrow Biopsy, Trephine Biopsy

\section{Introduction}

BM examination is a valuable diagnostic tool in various hematologic and non-hematologic conditions, though a good percentage of them are reflected in the peripheral blood. Bone marrow Aspirate(BMA) and bone marrow trephine biopsy (BMB) are the two diagnostic procedures done to evaluate the disease process. Though both the procedures done on the same day are complementary to each other, discrepancies do occur. The occurrence of a dry tap or blood tap in certain disease conditions and technical default, lead to faulty diagnosis of the BMA in certain disease conditions. The BMA procedure is relatively easier and provides better cellular morphology and cell count; therefore it is always done by the clinician. In view of the pain and a relatively complex procedure BMB is often neglected. This study is aimed to statistically analyze the diagnostic value of both the procedures and the lacuna observed when any one of the procedure alone is performed.

\section{Materials and Methods}

A retrospective laboratory record based analysis was done in the Department of Pathology on the bone marrow specimens reported during a period of 5 years (Jan.2011 - Dec.2015).All the bone marrow investigations with simultaneous BMA and $\mathrm{BMB}$ done during the period of study were included. Institution ethics committee approved the study. All the BMA has been stained with Leishman stain and May Grunwald Giemsa stain and Perl's stain for iron. All the BMB were fixed in $10 \%$ buffered formalin, decalcified in $10 \%$ formic acid and stained with hematoxylin and eosin after decalcification. Reticulin and iron stains are routinely done and other special stains and immunohistochemistry (IHC) were done whenever necessary. The reports had been released with the consensus of three pathologists.

The BMB reports were categorized into 12 categories as shown in Table 1. The numbers of BMA reports correlating with $\mathrm{BMB}$ were tabulated. The non concordant reports were also tabulated according to the categories in Table 2.

Descriptive statistical analysis was done using GNU PSPPO.8.4 for correlation of BMA and BMB diagnosis. The causes for poor correlation were discussed in comparison with previous studies.

\section{Results}

During the five years $934 \mathrm{BMB}$ and 1379 BMA were reported. All the BMB were preceded by BMA at the same time and usually the same site as suggested by Islam A. ${ }^{[1]}$ 
The age group of the patients ranged from 14 days of life to 82 years. The male: female ratio was $1.4: 1$. The diagnostic sensitivity of BMB was 94.74\% (811 cases) and BMA was $86.14 \%$ (584 cases). BMB and BMA reports were complementary to each other in $497(53.21 \%)$ cases. BMB alone was diagnostic in $314(33.61 \%)$ cases and BMA alone was diagnostic in $87(9.31 \%)$ cases. Inadequate material for diagnosis was noted in $78(8.35 \%)$ of BMB cases and $256(27.41 \%)$ of BMA cases. Table 1. shows the number of cases in each category according to BMB reports and the sensitivity and false negativity with BMA. Table 2 . shows the spectrum of BMA in comparison with BMB reports when there was negative correlation. Good positive correlation was noted in cases of immune thrombocytopenic purpura $(75.68 \%)$, multiple myeloma $(70.9 \%)$, anemia (69.37\%), reactive marrow $(67.57 \%)$ and chronic lymphocytic leukemia $(60 \%)$. Poor correlation was seen in granulomatous inflammation $(10 \%)$ myeloproliferative neoplasms (25\%) metastasis (27.27\%), lymphoma (29.41\%) and myelodysplastic syndrome $(32.43 \%)$.

BMA was the tool in diagnosis of $45(4.82 \%)$ cases reported as normal in BMB and 41 (4.39\%) cases where the BMB was inadequate for evaluation. A case of hypocellular MDS was diagnosed by BMA.

\section{Discussion}

Bone marrow examination is a valuable investigation in hematologic and various non-hematologic disorders. This study was conducted to conclude whether BMA and BMB should be advocated at the same time which is still a controversy.

The diagnostic sensitivity of BMB was $86.83 \%$ including inadequate samples and $94.74 \%$ excluding them, which is almost similar to previous studies by Chandra et. al (99.2\%) and Ayadayesh M.H. et. al (98.8\%). ${ }^{[2,3]}$ The BMA was diagnostic in $62.5 \%$ when inadequate smears were included and $86.14 \%$ excluding them. The diagnostic accuracy was slightly higher than the other studies. BMA and BMB were complementary to each other in $497(53.21 \%$ ) cases. $27.41 \%$ inadequate aspirates were responsible for the lower percentage than previous studies. ${ }^{[4,5]}$ Good positive correlation was observed with cases of immune thrombocytopenic purpura (ITP), anemia and reactive marrow among the non malignant conditions. The same observations were noted by Toi PC et. al. and Khan T.A. et al. ${ }^{[4,6]}$ Though erythroid hyperplasia and increased megakaryocytes were observed in anemia and ITP in the BMB, morphologic assessment was possible with BMA .Hypocellular

Table 1: No. of cases and correlation of BMA and BMB

\begin{tabular}{|c|c|c|c|c|c|c|c|}
\hline \multirow[t]{2}{*}{ S. No. } & \multirow[t]{2}{*}{ Disease } & \multicolumn{2}{|c|}{ BMB } & \multicolumn{2}{|c|}{$\begin{array}{c}\text { BMA - Diagnostic } \\
\text { sensitivity }\end{array}$} & \multicolumn{2}{|c|}{ BMA - False negative } \\
\hline & & Cases & $\%$ & Cases & $\%$ & Cases & $\%$ \\
\hline 1 & $\begin{array}{l}\text { Hypocellular } \\
\text { marrow }\end{array}$ & 170 & 18.2 & 60 & 35.3 & 110 & 64.7 \\
\hline 2 & ITP & 37 & 39.6 & 28 & 75.68 & 9 & 24.32 \\
\hline 3 & Acute leukemia & 147 & 15.7 & 83 & 56.46 & 64 & 43.53 \\
\hline 4 & MPN & 52 & 5.57 & 13 & 25 & 39 & 74.91 \\
\hline 5 & MDS & 37 & 3.96 & 12 & 32.43 & 25 & 67.58 \\
\hline 6 & MM & 38 & 4.07 & 27 & 70.9 & 11 & 28.95 \\
\hline 7 & Metastasis & 11 & 1.18 & 3 & 27.27 & 8 & 72.73 \\
\hline 8 & Anemia & 36 & 3.85 & 25 & 69.37 & 11 & 30.56 \\
\hline 9 & Inflammatory & 10 & 1.07 & 1 & 10 & 9 & 90 \\
\hline 10 & Lymphoma & 17 & 1.82 & 5 & 29.41 & 12 & 70.59 \\
\hline 11 & Normal/Reactive & 296 & 31.69 & 200 & 67.57 & 96 & 32.43 \\
\hline 12 & Inadequate & 78 & 8.35 & 37 & 47.44 & 41 & 52.56 \\
\hline 13 & CLL & 5 & 0.54 & 3 & 60 & 2 & 40 \\
\hline
\end{tabular}


Table 2: BMA reports which differed from BMB

\begin{tabular}{|c|c|c|c|c|c|}
\hline S. No. & Category - BMB & BMA & No. & $\%$ & False negative \\
\hline \multirow[t]{6}{*}{1} & \multirow[t]{6}{*}{ Hypo cellular marrow } & Inadequate & 65 & 38.23 & \multirow[t]{6}{*}{64.7} \\
\hline & & Normal & 35 & 20.59 & \\
\hline & & ITP & 2 & 1.18 & \\
\hline & & MDS & 1 & 0.59 & \\
\hline & & Anemia & 6 & 3.53 & \\
\hline & & Inflammation & 1 & 0.59 & \\
\hline \multirow[t]{3}{*}{2} & \multirow[t]{3}{*}{ ITP } & Inadequate & 3 & 8.11 & \multirow[t]{3}{*}{24.32} \\
\hline & & Normal & 4 & 10.8 & \\
\hline & & Hypocellular & 2 & 5.41 & \\
\hline \multirow[t]{5}{*}{3} & \multirow[t]{5}{*}{ Acute leukemia } & Inadequate & 55 & 37.41 & \multirow[t]{5}{*}{43.53} \\
\hline & & Normal & 3 & 2.04 & \\
\hline & & Hypocellular & 3 & 2.04 & \\
\hline & & Lymphoma & 2 & 1.36 & \\
\hline & & MDS & 1 & 0.68 & \\
\hline \multirow[t]{3}{*}{4} & \multirow[t]{3}{*}{ MPN } & Inadequate & 26 & 49.91 & \multirow[t]{3}{*}{74.91} \\
\hline & & Normal & 8 & 15.38 & \\
\hline & & Anemia & 5 & 9.62 & \\
\hline \multirow[t]{3}{*}{5} & \multirow[t]{3}{*}{ MDS } & Inadequate & 15 & 40.55 & \multirow[t]{3}{*}{67.58} \\
\hline & & Hypocellular & 3 & 8.11 & \\
\hline & & Anemia & 7 & 18.92 & \\
\hline 6 & MM & Inadequate & 11 & 28.95 & 28.95 \\
\hline 7 & Metastasis & Inadequate & 8 & 72.73 & 72.73 \\
\hline 8 & Anemia & Inadequate & 11 & 30.56 & 30.56 \\
\hline \multirow[t]{4}{*}{9} & \multirow[t]{4}{*}{ Granulomatous inflammation } & Inadequate & 3 & 30 & \multirow[t]{4}{*}{90} \\
\hline & & Normal & 4 & 40 & \\
\hline & & Hypocellular & 1 & 10 & \\
\hline & & Hemophagoatosis & 1 & 10 & \\
\hline \multirow[t]{3}{*}{10} & \multirow[t]{3}{*}{ Lymphoma } & Inadequate & 8 & 47.06 & \multirow[t]{3}{*}{70.59} \\
\hline & & Normal & 3 & 17.65 & \\
\hline & & Acute Leukemia & 1 & 5.88 & \\
\hline \multirow[t]{8}{*}{11} & \multirow[t]{8}{*}{ Normal/Reactive } & Inadequate & 51 & 17.23 & \multirow[t]{8}{*}{32.43} \\
\hline & & Inflammatory & 2 & 0.66 & \\
\hline & & Chediak-Higashi & 1 & 0.33 & \\
\hline & & Hemophagocytosis & 2 & 0.66 & \\
\hline & & Lympho Proliferative disorder & 1 & 0.33 & \\
\hline & & Hypocellular & 14 & 44.73 & \\
\hline & & ITP & 5 & 1.69 & \\
\hline & & Anemia & 20 & 76.76 & \\
\hline 12 & Inadequate & Normal & 26 & 33.33 & 52.56 \\
\hline & & Acute Leukemia & 8 & 10.26 & \\
\hline & & Anemia & 4 & 5.13 & \\
\hline & & ITP & 2 & 2.56 & \\
\hline & & MDS & 1 & 1.28 & \\
\hline
\end{tabular}


marrow and chronic granulomatous inflammation were better diagnosed with BMB. This had been well stated by Bain BJ. ${ }^{7}$

Among the hematologic malignancies multiple myeloma (MM) had good positive correlation of $70.9 \%$ similar to earlier studies. ${ }^{8}$ Also BMB provided a platform to perform IHC to assess the clonality. BMA was helpful in diagnosing $56.46 \%$ of acute leukemia diagnosed by BMB. The major cause for lack of confirmatory diagnosis was inadequate aspirate $(37.41 \%)$. Though BMB was considered unnecessary for the diagnosis of acute leukemia if the aspirate is good, a dry tap is often noticed due to packed marrow which may not permit BMA diagnosis as well as adequate samples for flowcytometry also. BMB was the only conclusive diagnostic tool in those cases and categorization can be done with IHC. The diagnostic utility of BMA in myeloproliferative neoplasms(MPN) was low since all the cases were myelofibrosis which yielded a dry tap. BMB was superior to BMA in diagnosing lymphoma. Our observations were similar to Sitalakshmi et al and Nanda et al and are in contradiction to Mahajan et al.$^{9,10,5}$ Moderate positive correlation was noted in CLL. But $40 \%$ of cases were not diagnosed similar to Kaur et al. ${ }^{11}$ Though morphological characters of MDS were observed well in BMA, the diagnostic accuracy of BMB was superior as abnormal localization of immature myeloid precursors can be identified and IHC can be performed as stated by Winfield DA in his review papers ${ }^{12}$. The diagnostic accuracy of lymphoid infiltration in the BMA was only $30 \%$ lower than the study by Musolino et al. ${ }^{13}$ The non hematologic malignancies were diagnosed with better accuracy in BMB as observed by Mishra $\mathrm{P}$ et al. ${ }^{14}$ In cases of unknown primary site or unsuspected malignancy, BMB had been a tool for diagnosis and IHC.

Though BMA and BMB are complementary to one another, the diagnostic accuracy of BMB is more in granulomatous and infiltrative diseases. In acute leukemia when there is a packed marrow, BMA is often inadequate. However in our study, a few cases of Chediak-Higashi syndrome, hemophagocytosis and myelodysplastic syndrome(MDS) could be diagnosed by BMA when there was inadequate BMB.

\section{Conclusion}

The present study indicates that BMA and BMB are highly valuable diagnostic procedures in hematological and non hematologic disorders as well as in malignant and non malignant conditions. Though both the procedures were complimentary to each other, inadequate aspirates due to disease conditions and faulty techniques are major drawbacks. BMB provided a histologic overview and was more accurate in diagnosing infiltrative conditions with an added advantage of performing IHC. Though painstaking, it is preferable to perform both the procedures simultaneously for a more conclusive diagnosis.

\section{Acknowledgement}

The authors wish to thank Dr. Suresh Varadarajan, Associate Professor, Community Medicine, SRMC \& RI for his contribution towards statistical analysis and Mrs. Uma Lakshmi, Clinical Instructor for technical assistance.

\section{References}

1. Islam A. Bone marrow aspiration before bone marrow core biopsy using the same bone marrow biopsy needle: a good or bad practice?. J ClinPathol. 2007;60:212-215.

2. Chandra $\mathrm{S}, \mathrm{Chandra} \mathrm{H}$. Comparison of bone marrow aspirate cytology, touch imprint cytology and trephine biopsy for bone marrow evaluation. Hematol Rep. 2011;3:65-68.

3. Aljadayeh MH, Saidat SD, Kamal N, Telfah A. Comparative Evaluation between Bone Marrow Aspirate and Biopsy Morphologic Findings, Experience At King Hussein Medical Center. JRMS. 2015;22:18-22.

4. Toi PC, Varghese RB, Rai R. Comparative evaluation of simultaneous bone marrow aspiration and bone marrow biopsy: an institutional experience. Indian J Hematol Blood Transfus. 2010;26:41-44.

5. Mahajan V, Kaushal V, Thakur S, Kaushik R. A comparative study of bone marrow aspiration and bone marrow biopsy in haematological and non haematological disorders-An institutional experience. JIACM. 2013;14;133-135

6. Khan TA, Khan IA, Mahmood K. Diagnostic role of bone marrow aspiration and trephine biopsy in haematological practice. J Postgrad Med Institute. 2014;28:217-221.

7. Bain BJ. Bone marrow trephine biopsy. J ClinPathol. 2001; 54:737-742.

8. Štifter S, Babarović E, Valković T, Seili-Bekafigo I, Štemberger C, Načinović A, Lučin K, Jonjić N. Combined evaluation of bone marrow aspirate and biopsy is superior in the prognosis of multiple myeloma. Diagnpathol. 2010; 5:30-37.

9. Sitalakshmi S, Srikrishna A, Devi S, Damodar P, Alexander B. The diagnostic utility of bone marrow trephine biopsies. Indian J PatholMicrobiol. 2005; 48:173176.

10. Nanda A, Basu S, Marwaha N. Bone marrow trephine biopsy as an adjunct to bone marrow aspiration. $\mathrm{J}$ Assoc Physicians India. 2002; 50:893-895.

11. Kaur M, Rana AP, Kapoor S, Puri A. Diagnostic Value of Bone Marrow Aspiration and Biopsy in Routine Hematology Practice. JCDR. 2014;8:FC13-16 
12. Winfield DA, Polacarz SV. Bone marrow histology. 3: Value of bone marrow core biopsy in acute leukaemia, myelodysplastic syndromes, and chronic myeloid leukaemia. J ClinPathol. 1992;45:855-859

13. Muslino A, Guazzi A, Nizzoli R, Panebianco M, Mancini C, Ardizzoni A. Accuracy and relative value of bone marrow aspiration in the detection of lymphoid infiltration in non - Hodgkin lymphoma. Tumori.2010;96;24-27.

14. Mishra P, Das S, Kar R, Jacob SE, Basu D. Nonhaematopoietic malignancies metastasing to the bone marrow: A 5 year record-based descriptive study from a tertiary care centre in South India. India. Indian J Cancer. 2014;51:30-34 Indian J Cancer. 2014;51:30-34

*Corresponding author:

Dr Febe Renjitha Suman, A 5 Staff quarters, SRU Campus, Porur, Chennai-600116, Tamilnadu, India.

Phone: +91 9994081470

Email: febemd@gmail.com

Date of Submission : 12.07.2017

Date of Acceptance : 03.10.2017

Financial or other Competing Interests: None.

Date of Publication : 27.01.2018 